УДК $550.834 ; 621.373$

\title{
ИССЛЕДОВАНИЕ РАБОТЫ НИЗКОВОЛЬТНОГО УДАРНОГО ГЕНЕРАТОРА В УСТРОЙСТВЕ ЭЛЕКТРОГИДРАВЛИЧЕСКОГО ВОЗДЕЙСТВИЯ ДЛЯ МАЛОГЛУБИННОЙ СЕЙСМОРАЗВЕДКИ
}

\section{Пустынников Сергей Владимирович',}

pustynnikov@tpu.ru

\author{
Носов Геннадий Васильевич', \\ nosov@tpu.ru
}

\author{
Хан Вей², \\ whan@jlu.edu.cn

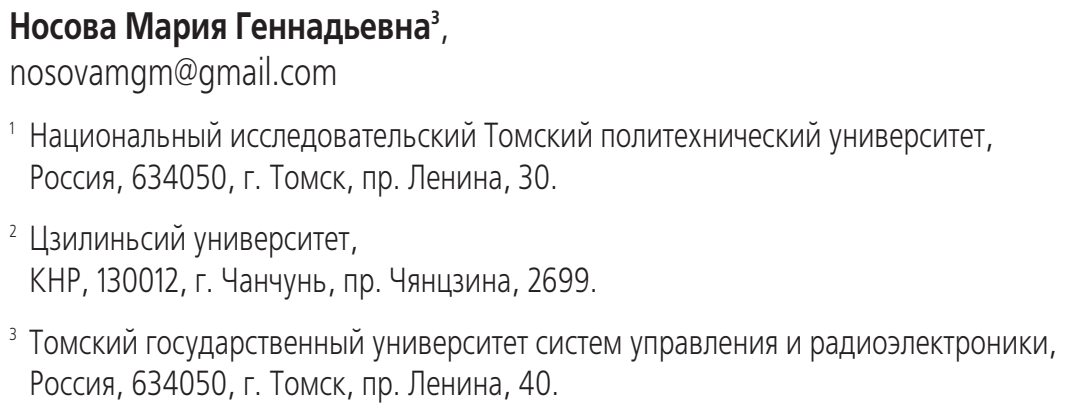

Актуальность исследования обусловлена необходимостью разработки новых источников мощных импульсов тока для работы на электрогидравлический излучатель с раздвигающимися электродами, который может применяться для возбуждения сейсмических волн в невзрывной сейсморазведке.

Цель: провести расчетные и экспериментальные исследования работы низковольтного ударного генератора на дугу в воде, инициированную раздвигающимися электродами в режиме одиночных импульсов и при получении серии импульсов давления. Провести оптимизацию параметров ударного генератора и электрогидравлического излучателя с целью получения максимального импульса давления.

объекты: низковольтный ударный генератор, выполненный в габаритах кранового асинхронного двигателя МТН-612, подключенный к электрогидравлическому излучателю с раздвигающимися электродами.

Методы: согласование параметров ударного генератора и дугового разряда методом планирования эксперимента с помощью математической модели.

Результаты. Проведены лабораторные исследования работы низковольтного ударного генератора на дугу в воде, инициированную раздвигающимися электродами в режиме одиночных импульсов и при получении серии импульсов. Методом планирования эксперимента получена математическая модель работы ударного генератора на дугу в воде, инициированную раздвигающимися электродами в режиме одиночных импульсов, получены уравнения регрессии для выходных параметров дугового разряда, проведена оптимизация параметров ударного генератора и электрогидравлического излучателя, что позволяет получить максимальный импульс давления для проведения малоглубинной сейсморазведки. Полученный амплитудно-частотный спектр импульса давления показал, что основная энергия импульса давления лежит в среднечастотном диапазоне от 50 до 100 Гц, что обеспечивает высокий сейсмический КПД для проведения малоглубинной сейсморазведки.

\section{Ключевые слова:}

Ударный генератор, электрогидравлический излучатель, раздвигающиеся электроды, математическая модель, осциллограмма, сейсморазведка.

\section{Введение}

В настоящее время электрогидравлический эффект имеет широкое применение в промышленных технологиях [1-9]:

- различные виды очистки;

- снятие внутренних напряжений;

- штамповка;

- сварка;

- электрогидравлические молоты и вибраторы;

- электрогидравлические насосы;

- дробление и измельчение;

- (де)эмульгация;

- обеззараживание;

- медицинские технологии;

- морская и наземная сейсморазведка.
Создание сейсмических волн при помощи взрыва заряда взрывчатого вещества на поверхности или в скважине традиционно применяется в сейсморазведочный работах. Однако такой метод имеет ограниченное применение и не может использоваться в местах поселений, вблизи мостов, линий электропередач, железных дорог и т. д. Кроме того, взрывной метод наносит значительный вред окружающей среде [10]. В настоящее время получила развитие невзрывная сейсморазведка, позволяющая упростить производство работ, обеспечить безопасное их проведение для обслуживающего персонала и окружающей среды. $К$ таким методам относится возбуждение сейсмических волн с помощью вибрационных источников, импульсных нев- 
зрывных источников, использующих энергию выхлопа в воду сильно сжатого воздуха, а также электрогидравлических источников, которые позволяют возбуждать сейсмические волны при помощи электрического разряда в воде, вызывающего импульсное преобразование электрической энергии в энергию упругой волны давления [11].

По месту проведения сейсморазведка подразделяется на наземную (полевую), акваториальную (морскую), скважинную и подземную, а по частотам колебаний используемых упругих волн можно выделить высокочастотную (частоты свыше 100 Гц), среднечастотную (частоты в несколько десятков Гц) и низкочастотную (частоты менее 10 Гц) сейсморазведку. Чем выше частота упругих волн, тем больше их затухание и меньше глубинность сейсморазведки [12].

Существующие в настоящее время установки для электрогидравлического возбуждения сейсмических колебаний используют в качестве накопителей импульсной энергии конденсаторные батареи. В условиях массогабаритных ограничений, накладываемых требованиями автономности и транспортабельности, энергия конденсаторной батареи относительно невысока и составляет от 5 до 20 кДж при напряжении от 30 до 70 кВ [13, 14]. K недостаткам таких установок следует отнести наличие высокого напряжения, снижающего безопасность работ, и высокочастотный спектр возбуждаемых волн давления, обусловливающий низкий сейсмический КПД.

В устройствах электрогидравлического воздействия, требующих получения импульсов давления невысокой амплитуды (до $10 \mathrm{MПа)} \mathrm{и} \mathrm{большой} \mathrm{дли-}$ тельности (5-10 мс), целесообразным является применение электромашинных источников импульсов тока (ударных генераторов). Однако имеются трудности, связанные с инициированием электрического разряда вследствие невысокого напряжения статорной обмотки (до 1 кВ), уровень которого определяется наиболее эффективным использованием активного объёма генератора.

Впервые исследования применения ударного генератора для возбуждения сейсмически колебаний были выполнены в Томском политехническом институте [15]. Инициирование канала разряда осуществляется предварительным разрядом высоковольтной конденсаторной батареи или при низком напряжении - взрывающимися проволочками. В первом случае требуется защита низковольтного ударного генератора от высокого напряжения, что значительно усложняет схему устройства, во втором случае работа электрогидравлического устройства возможна только в режиме единичных воздействий, т. к. взрывающуюся проволочку необходимо периодически заменять. Амплитуда импульса давления определяется величиной тока в момент взрыва проводника. Установлено, что максимальный гидродинамический эффект достигается в момент, близкий к максимуму тока короткого замыкания ударного генератора, при этом длительность импульса тока в нагрузке по результатам эксперимента составляла порядка $t_{\text {имп }} \approx 5-10$ мс $[16,17]$.

Основное внимание в статье уделяется проведённым в Томском политехническом университете исследованиям работы низковольтного ударного генератора, выполненного в габаритах асинхронного двигателя МТН-612 на электрогидравлический излучатель, состоящий из предварительно замкнутых электродов, которые расходятся под действием электродинамических усилий при протекании тока ударного генератора и растягивают электрическую дугу, загорающуюся между электродами [18].

\section{Постановка задачи}

Силовая схема лабораторного устройства для работы ударного генератора на дугу с изменяющейся длиной межэлектродного промежутка показана на рис. 1.

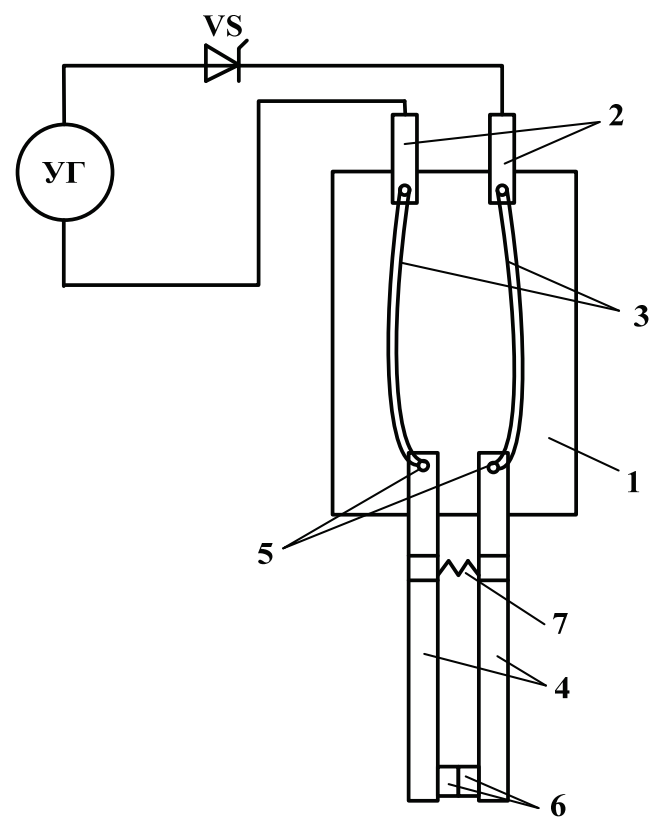

Рис. 1. Силовая схема лабораторного устройства: УГ-ударный генератор; VS - тиристорный комлутатор; 1 - основание; 2 - токоподводы; 3 - гибкие проводники; 4 - подвижные электроды; 5 - шарниры; 6 - контакты; 7 - возвратная пружина

Fig. 1. Power circuit of the laboratory device: УГ is the shock generator; VS is the thyristor switch; 1 is the circuit board; 2 is the hollow conductor; 3 is the flexible conductor; 4 are the moving electrodes; 5 is the gimbal; 6 are the power contacts; 7 is the return spring

Ударный генератор - УГ через тиристорный коммутатор - VS подключён к электрогидравлическому излучателю, помещённому в бак с водопроводной водой. На текстолитовом основании - 1 электрогидравлического излучателя размещены токоподводы - 2 к гибким проводникам - 3 , которые подключены к подвижным электродам - 4, вращающимся на шарнирах - 5. Подвижные электроды -4 имеют контакты - 6, прижатые друг к другу возвратной пружиной - 7. Ударный генератор УГ 
приводится во вращение и возбуждается до номинальной ЭДС. После включения тиристорного коммутатора - VS ток короткого замыкания идёт через токоподводы - 2, гибкие проводники - 3, через медные подвижные электроды - 4 и замкнутые контакты - 6. Возникающие электродинамические усилия, преодолевая силу возвратной пружины - 7, раздвигают подвижные электроды - 4, между контактами - 6 загорается электрическая дуга, формирующая упругую волну давления. После прохождения положительного импульса тока дуга гаснет, и подвижные электроды - 4 возвращаются в исходное состояние силой возвратной пружины -7 .

Задачами данной статьи являются экспериментальные исследования лабораторной модели устройства, создание математической модели работы ударного генератора на дугу, инициированную раздвигающимися электродами, проведение расчётов с помощью математической модели с целью оптимизации параметров электрогидравлического излучателя для получения максимального импульса волны давления, применяемого в малоглубинной сесморазведке.

\section{Экспериментальные исследования}

Лабораторные исследования физической модели электрогидравлического излучателя (рис. 2) были проведены с помощью ударного генератора, выполненного в габаритах асинхронного двигателя МТН-612 (рис. 3). Ударный генератор имеет следующие параметры: действующее напряжение статорной обмотки $U=280$ В, ударный ток внезапного короткого замыкания $I_{\text {уд }}=28 \kappa \mathrm{A}$, частота вращения ротора $n=3000$ об/мин, что соответствует частоте ЭДС $f=50$ Гц. Электрогидравлический излучатель погружался в бак с водопроводной водой на глубину $0,5 \mathrm{~m}$.

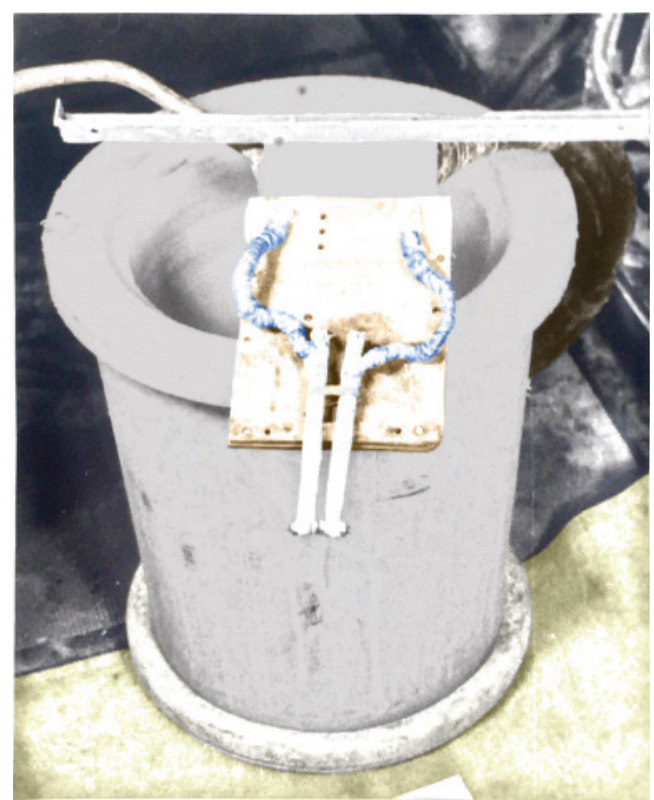

Рис. 2. Физическая модель электрогидравлического излучателя

Fig. 2. Physical model of electrohydraulic source

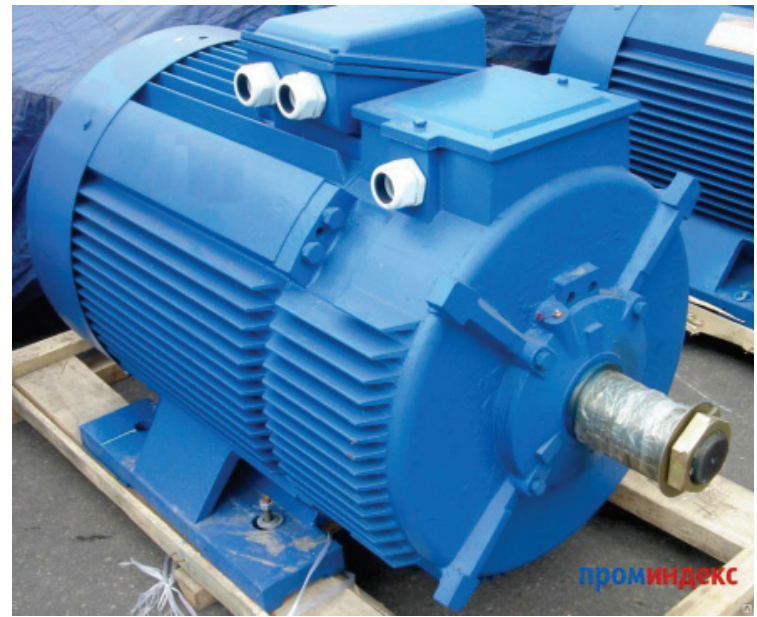

Pис. 3. Ударный генератор в габаритах асинхронного двигателя MTH-612

Fig. 3. Shock generator in the dimensions of the asynchronous motor MTN-612

Для регистрации угла поворота подвижных электродов вместо одного из шарниров, на которых поворачиваются подвижные электроды, поставлен движок переменного сопротивления СПО. Изменение падения напряжения на сопротивлении фиксируется осциллографом. На рис. 4 показана типичная осциллограмма тока дуги $I$, напряжения дуги $U$ и угла поворота раздвигающихся электродов $\varphi$. Длина подвижных электродов $L=0,14 \mathrm{~m}$, максимальная длина дуги $l=0,08 \mathrm{M}$, угол включения тиристорного коммутатора $\alpha=0^{\circ}$.

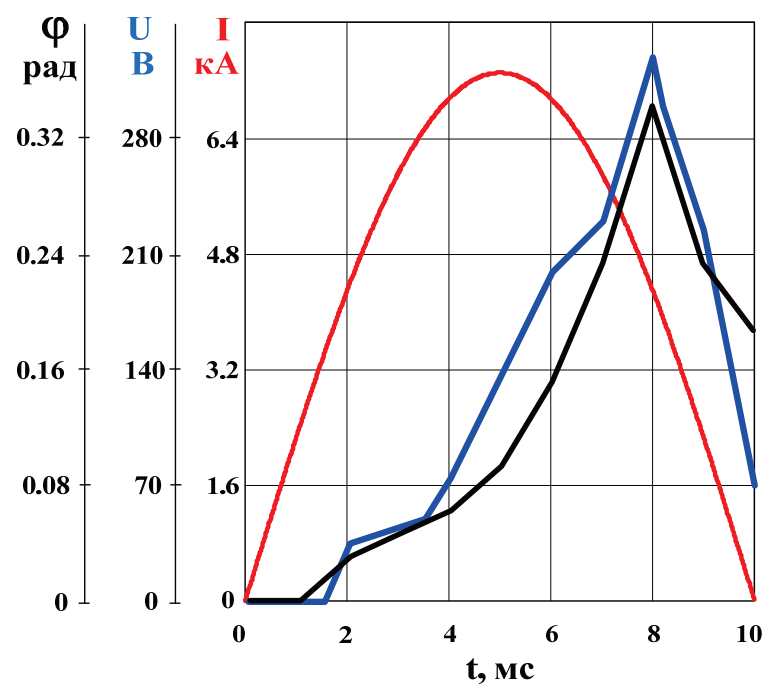

Puс. 4. Осииллогралма тока дуги I, напряжения дуги U, угла поворота раздвигающихся электродов $\varphi$

Fig. 4. Oscillogram of arc current $I$, arc voltage $U$, rotation angle of moving electrodes $\varphi$

На рис. 5 приведены временные зависимости сопротивления $r$, мощности $p$ и энергии $w$ дуги при тех же параметрах электрогидравлического излучателя. Характерной особенностью кривой сопротивления $r$ является то, что в начальный момент 

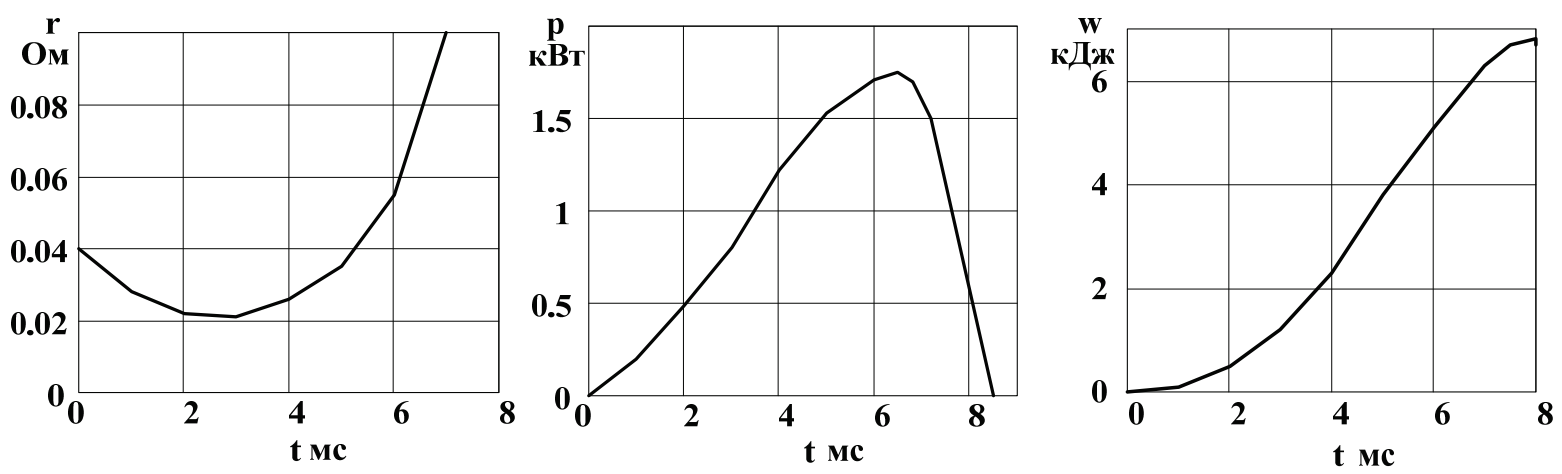

Puс. 5. Сопротивление r, мощность ри энергия $w$ дуги

Fig. 5. Resistance $r$, power $p$ and energy $w$ of the arc

времени сопротивление межэлектродного промежутка определяется сопротивлением окисной плёнки поверхности электродов. Для стальных электродов оно составляет порядка 0,04 Ом. После момента начала движения электродов кривая изменения сопротивления дуги является типичной для дугового разряда ударного генератора.

При лабораторных испытаниях устройства была исследована возможность получения серии импульсов, состоящих из 5-10 подводных взрывов с частотой следования 25 Гц. На рис. 6 приведены осциллограммы тока $I$ и напряжения дуги $U$ при непрерывной работе ударного генератора на раздвигающиеся электроды. Из осциллограмм следует, что амплитуда импульсов тока снижается вследствие уменьшения кинетической энергии ротора. Материал контактов подвижных электродов находится под воздействием высокой температуры горения дуги до $7000{ }^{\circ} \mathrm{C}$, поэтому при длительном горении дуги до 10 мс наблюдается эрозия электродов. При испытаниях применялся различный материал контактов: вольфрам, сталь, углеграфит. Углеграфит разрушался после 1-2 взрывов, сталь выдерживала 10-15 взрывов, вольфрам 50-70 взрывов.

\section{Теоретические исследования}

Проведённые экспериментальные исследования позволили скорректировать формулу Теплера для расчёта сопротивления дугового канала для стальных электродов [19]:

$$
r=k \cdot l \cdot e^{\sqrt{\delta t}} / \int_{0}^{t} i \cdot d t,
$$

где $k=0,02 \mathrm{~B} \cdot \mathrm{c} / \mathrm{м}$ - постоянный коэффициент; $l$ длина дуги, м; $t$ - время, с.

Коэффициент $\delta$ скорректирован при обработке осциллограмм и определяется по формуле:

$$
\delta=a \cdot t^{b}+c
$$

где коэффициенты $a=3,41 / \mathrm{c}, b=-1,344, c=4700$, подобраны методом наименьших квадратов.

Формулы (1), (2) позволяют определить сопротивление дуги при работе ударного генератора для известного значения длины дуги в данный момент времени с достаточной для инженерных расчётов точностью.



Pис. 6. Осциллогралмы тока дуги I при непрерывной работе ударного генератора на раздвигающиеся электроды

Fig. 6. Oscillograms of arc current I during continuous operation of the shock generator on moving electrodes 
Электромагнитная энергия, передаваемая генератором в электрическую дугу, преобразуется в механическую энергию парогазового пузыря. При изменении геометрических размеров системы подвижных электродов в значительной степени изменяются параметры разряда: амплитуда тока $I_{m}$, длительность горения дуги $t_{d}$, величина вводимой в дугу энергии $w$, амплитуда импульса давления $N_{m}$.

Для расчёта амплитуды импульса давления применялась формула, приведённая в [11]:

$$
N_{m}=\frac{k_{p} \cdot H / l}{\sqrt{H}} \cdot \rho_{0}^{3 / 8} \cdot\left[\frac{w}{l}\right]^{5 / 8} \cdot t_{d}^{-3 / 4},
$$

здесь $H$, м - расстояние от оси межэлектродного промежутка до точки измерения, с учётом диаметра скважины, в которой должен находиться электрогидравлический излучатель: $H=0,1 \mathrm{~m} ; k_{p}-$ коэффициент, зависящий от соотношения $H / l$, для $H / l$ от 0 до $2,6 k_{p}=0,46 ; \rho_{0}=103 \kappa \Gamma / \mathrm{m}^{3}$ - объёмная плотность водопроводной воды.

Согласование параметров ударного генератора и дугового разряда осуществлялось методом планирования эксперимента с помощью математической модели [20]. Система дифференциальных уравнений, описывающих переходный процесс в обмотках ударного генератора и в цепи нагрузки, имеет вид [21]:

$$
\left\{\begin{array}{l}
d \psi_{a} / d t=-\left(r_{a}+r\right) \cdot i \\
d \psi_{f d} / d t=U_{f}-r_{f d} \cdot i_{f d}
\end{array} ;\right.
$$

где $\psi_{a}, \psi_{f d}, \psi_{y d}, \psi_{y q}$ - потокосцепления статорной, возбуждения, продольной и поперечной демпферной обмоток; $r_{a}, r_{f d}, r_{y d}, r_{y q}$ - активные сопротивления обмоток; $r$ - нелинейное сопротивление нагрузки (дугового канала); $i, i_{f d}, i_{y d}, i_{y q}$ - токи, протекающие по обмоткам статора и ротора.

Параметры сопротивлений ударного генератора, выполненного в габаритах асинхронного двигателя МТН-612, приведены в табл. 1.

Решение системы уравнений (3) проведено численным методом последовательных интервалов, что позволило создать математическую модель работы ударного генератора на дугу в воде с изменяющимся межэлектродным промежутком. Работа ударного генератора на электрогидравлический излучатель с раздвигающимися электродами описывается рототабельным планом второго порядка типа $2^{3}$. Значение уровней переменных: длины подвижных электродов $L$, конечного значения длины дуги $l$, угла включения генератора $\alpha$ приведены в табл. 2 .

Границы изменения длины подвижных электродов $L$, максимальной длины дуги $l$ и угла включения $\alpha$ охватывают оптимальный диапазон, полученный при проведении экспериментальных исследований. Кроме того, значение $l=0,07$ м при размещении электрогидравлического излучателя в скважине с водой ограничивается диаметром скважины, который не превышает 0,1-0,12 м.
Таблица 1. Параметры сопротивлений ударного генератора

\begin{tabular}{|c|c|c|}
\hline $\begin{array}{l}\text { Наименование } \\
\text { Name }\end{array}$ & 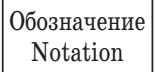 & $\begin{array}{c}\text { Величина (Ом) } \\
\text { Value (Ohm) }\end{array}$ \\
\hline $\begin{array}{l}\text { Активное сопротивление } \\
\text { обмотки статора } \\
\text { Active resistance of the stator winding }\end{array}$ & $r_{a}$ & 0,00187 \\
\hline \begin{tabular}{|l|} 
Активное сопротивление обмотки \\
возбуждения \\
Field resistance \\
\end{tabular} & $r_{f d}$ & 0,0878 \\
\hline $\begin{array}{l}\text { Активное сопротивление демпферной } \\
\text { обмотки } \\
\text { Quadrature axis amortisseur resistance } \\
\end{array}$ & $r_{y d}=r_{y q}$ & 0,000076 \\
\hline \begin{tabular}{|l|} 
Индуктивное сопротивление \\
рассеяния статора \\
Armature leakage reactance \\
\end{tabular} & $x_{\sigma a}$ & 0,012 \\
\hline \begin{tabular}{|l|} 
Индуктивное сопротивление \\
рассеяния обмотки возбуждения \\
Field leakage reactance \\
\end{tabular} & $x_{\sigma f d}$ & 0,872 \\
\hline \begin{tabular}{|l|} 
Индуктивное сопротивление \\
рассеяния демпферной обмотки \\
Direct axis amortisseur reactance \\
\end{tabular} & $x_{\sigma D d}=x_{\sigma D q}$ & 0,00049 \\
\hline \begin{tabular}{|l} 
Индуктивное сопротивление \\
взаимной индукции \\
Direct axis armature reactance \\
\end{tabular} & $x_{a d}=x_{a q}$ & 0,12 \\
\hline \begin{tabular}{|l|} 
Сверхпереходное индуктивное \\
сопротивление \\
Direct axis subtransient reactance \\
\end{tabular} & $x_{d}^{\prime}$ & 0,0121 \\
\hline $\begin{array}{l}\text { Ударное индуктивное сопротивление } \\
\text { Quadrature-axis subtransient impedance }\end{array}$ & $x_{y d}$ & 0,0249 \\
\hline
\end{tabular}

Table 1. Parameters of resistance of a shock generator

Таблица 2. Значения уровней переменных

Table 2. Variable level values

\begin{tabular}{|c|c|c|c|c|c|c|}
\hline \multirow{2}{*}{$\begin{array}{c}\text { Переменные } \\
\text { Variables }\end{array}$} & \multirow{2}{*}{$\begin{array}{c}\text { Обозначение } \\
\text { Notation }\end{array}$} & \multicolumn{5}{|c|}{$\begin{array}{c}\text { Значение уровней переменных } \\
\text { Value of the levels of variables }\end{array}$} \\
\cline { 3 - 7 } & & $-\varphi$ & -1 & 0 & +1 & $+\varphi$ \\
\hline$L$ (м) & $x_{1}$ & 0,1 & 0,14 & 0,18 & 0,22 & 0,26 \\
\hline$l$ (м) & $x_{2}$ & 0,03 & 0,04 & 0,05 & 0,06 & 0,07 \\
\hline$\alpha$ (град) & $x_{3}$ & 0 & 20 & 40 & 60 & 80 \\
\hline
\end{tabular}

Матрица планирования и результаты расчётов выходных параметров: амплитуды тока дуги $I_{m}$, энергии дуги $w$, длительности горения дуги $t_{d}$, средней мощности дуги $P_{d}=w / t_{d}$, амплитуды импульса давления $N_{m}$ представлены в табл. 3 .

После исключения членов с незначимыми коэффициентами проведён анализ уравнений на адекватность с помощью критерия Фишера. Адекватные уравнения регрессии для значений выходных параметров имеют вид:

$$
\left\{\begin{aligned}
I_{m} & =7,916-38,6 \cdot L-27,8 \cdot l- \\
& -0,00935 \cdot \alpha+13,5 \cdot l^{2}-0,005568 \cdot \alpha^{2} ; \\
w & =10,32-23,2 \cdot L+16,4 \cdot l- \\
& -0,0413 \cdot \alpha-0,00932 \cdot \alpha^{2} ; \\
t_{d} & =9,12-16,4 \cdot l-0,00842 \cdot \alpha-0,003348 \cdot \alpha^{2} ; \\
N_{m} & =13,78-147 \cdot l-0,0285 \cdot \alpha+ \\
& +29 \cdot l^{2}-0,001 \cdot \alpha^{2} ; \\
P_{d} & =1,13-1,93 \cdot L+3,75 \cdot l- \\
& -0,00368 \cdot \alpha-0,000102 \cdot \alpha^{2} .
\end{aligned}\right.
$$


Таблица 3. Матрица планирования и результаты расчётов выходных параметров

Table 3. $\quad$ Planning matrix and the results of calculations of output parameters

\begin{tabular}{|c|c|c|c|c|c|c|c|c|c|c|c|c|c|c|}
\hline \multirow{2}{*}{$x_{0}$} & \multirow{2}{*}{$x_{1}$} & \multirow{2}{*}{$x_{2}$} & \multirow{2}{*}{$x_{3}$} & \multirow{2}{*}{$x_{1}^{2}$} & \multirow{2}{*}{$x_{2}^{2}$} & \multirow{2}{*}{$x_{3}^{2}$} & \multirow{2}{*}{$x_{1} * x_{2}$} & \multirow{2}{*}{$x_{1} * x_{3}$} & \multirow{2}{*}{$x_{2} * x_{3}$} & $I_{m} \cdot 10^{3}$ & $w \cdot 10^{3}$ & $t_{d} \cdot 10^{-3}$ & $N_{m}$ & $P_{d} \cdot 10^{6}$ \\
\hline & & & & & & & & & & $\mathrm{A} / \mathrm{A}$ & Дж/J & $\mathrm{c} / \mathrm{s}$ & atm/atm & $\mathrm{BT} / \mathrm{Wt}$ \\
\hline+ & - & - & - & + & + & + & + & + & + & 9,05 & 12,82 & 9,68 & 17,35 & 1,324 \\
\hline+ & + & - & - & + & + & + & - & - & + & 8,56 & 12,26 & 9,63 & 16,95 & 1,274 \\
\hline+ & - & + & - & + & + & + & - & + & - & 8,5 & 12,87 & 9,4 & 13,81 & 1,37 \\
\hline+ & + & + & - & + & + & + & + & - & - & 7,58 & 12,1 & 9,3 & 13,4 & 1,3 \\
\hline+ & - & - & + & + & + & + & + & - & - & 7,53 & 7,12 & 8,72 & 13 & 0,816 \\
\hline+ & + & - & + & + & + & + & - & + & - & 7,2 & 6,92 & 8,73 & 12,76 & 0,79 \\
\hline+ & - & + & + & + & + & + & - & - & + & 7,6 & 7,64 & 8,53 & 10,72 & 0,896 \\
\hline+ & + & + & + & + & + & + & + & + & + & 6,83 & 7,43 & 8,53 & 10,75 & 0,895 \\
\hline+ & $-\varphi$ & 0 & 0 & $\varphi^{2}$ & 0 & 0 & 0 & 0 & 0 & 9,08 & 10,86 & 9,2 & 14,15 & 1,18 \\
\hline+ & $+\varphi$ & 0 & 0 & $\varphi^{2}$ & 0 & 0 & 0 & 0 & 0 & 7,34 & 9,87 & 9 & 13,55 & 1,097 \\
\hline+ & 0 & $-\varphi$ & 0 & 0 & $\varphi^{2}$ & 0 & 0 & 0 & 0 & 8,97 & 9,67 & 9,47 & 17,71 & 1,027 \\
\hline+ & 0 & $+\varphi$ & 0 & 0 & $\varphi^{2}$ & 0 & 0 & 0 & 0 & 7,75 & 10,52 & 8,77 & 11,64 & 1,2 \\
\hline+ & 0 & 0 & $-\varphi$ & 0 & 0 & $\varphi^{2}$ & 0 & 0 & 0 & 8,16 & 12,94 & 9,6 & 15,28 & 1,348 \\
\hline+ & 0 & 0 & $+\varphi$ & 0 & 0 & $\varphi^{2}$ & 0 & 0 & 0 & 6,04 & 4,42 & 7,6 & 9,3 & 0,582 \\
\hline+ & 0 & 0 & 0 & 0 & 0 & 0 & 0 & 0 & 0 & 7,91 & 10,28 & 9,1 & 13,78 & 1,13 \\
\hline+ & 0 & 0 & 0 & 0 & 0 & 0 & 0 & 0 & 0 & 7,91 & 10,28 & 9,1 & 13,78 & 1,13 \\
\hline+ & 0 & 0 & 0 & 0 & 0 & 0 & 0 & 0 & 0 & 7,91 & 10,28 & 9,1 & 13,78 & 1,13 \\
\hline+ & 0 & 0 & 0 & 0 & 0 & 0 & 0 & 0 & 0 & 7,91 & 10,28 & 9,1 & 13,78 & 1,13 \\
\hline+ & 0 & 0 & 0 & 0 & 0 & 0 & 0 & 0 & 0 & 7,91 & 10,28 & 9,1 & 13,78 & 1,13 \\
\hline+ & 0 & 0 & 0 & 0 & 0 & 0 & 0 & 0 & 0 & 7,91 & 10,28 & 9,1 & 13,78 & 1,13 \\
\hline
\end{tabular}

Система уравнений (4) представляет математическую модель работы ударного генератора на дугу в воде с изменяющимся межэлектродным промежутком.

Для проведения сейсморазведки определяющим параметром является максимальный импульс давления $N_{m}$, развиваемый электрогидравлическим излучателем. Исследования, проведённые на математической модели, показывают, что в данном случае амплитуда импульса давления $N_{m}$ практически не зависит от длины подвижных электродов $L$, а определяется конечным значением длины дуги $l$ и углом включения генератора $\alpha$.

На рис. 7 приведены графические зависимости амплитуды импульса давления $N_{m}$ и средней мощности дуги $P_{d}$ от угла включения при различных значениях длины дуги $l$.

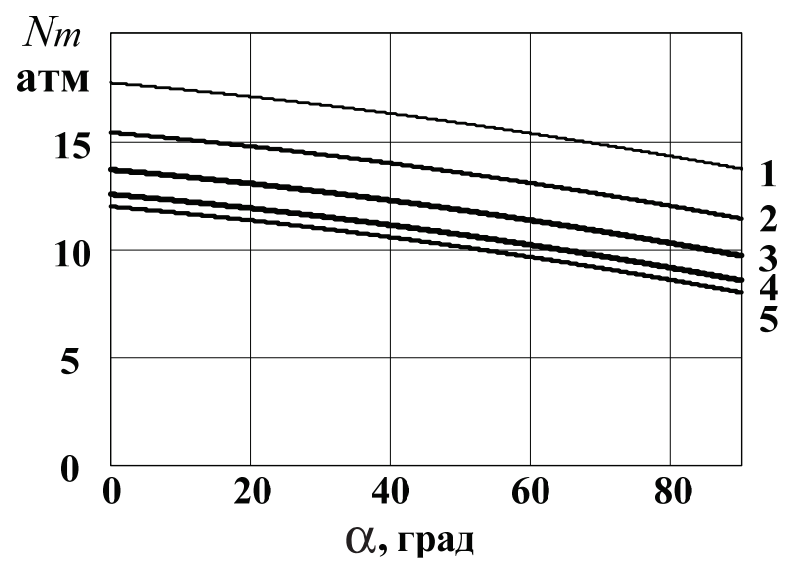

Проведённые исследования показали, что наилучшими значениями независимых параметров являются: длина подвижных электродов $L=0,1-0,26 \mathrm{м}$, конечное значение длины дуги $l=0,07 \mathrm{м}$, угол включения ударного генератора $\alpha=0^{\circ}$. Максимальное значение развиваемого импульса давления $N_{m}=17,71$ атм при средней мощности дуги $P_{d}=1,3$ МВт. Из исследований, проведённых в [17], следует, что форма кривой импульса давления повторяет форму кривой тока дуги. $\mathrm{C}$ помощью осциллограммы тока на рис. 7 получена формула разложения импульса давления с амплитудой $N_{m}=17,71$ атм в ряд Фурье для постоянной составляющей и трёх значимых гармоник:

$$
N_{m}(\omega t)=5,64+8,85 \cdot \sin (\omega t)+
$$

$+3,76 \cdot \sin \left(2 \omega t+90^{\circ}\right)+0,752 \cdot \sin \left(4 \omega t+90^{\circ}\right)$,

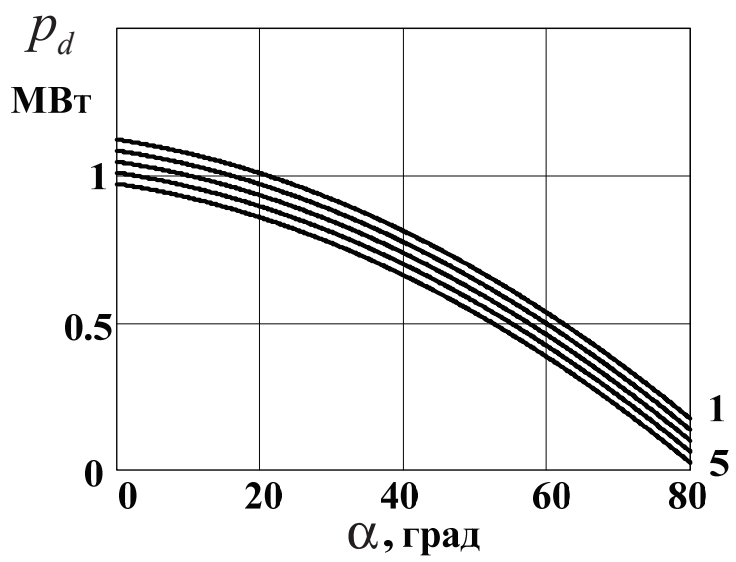

Рис. 7. Зависимость алплитуды илпульса давления $N_{m}$ и средней мощности дуги $P_{d}$ от угла включения $\alpha$ при L=0,14 л для длин дуги $l($ м ): 1 ) 0,$07 ; 2) 0,06 ; 3) 0,05 ; 4) 0,04 ; 5) 0,03$

Fig. 7. Dependence of the pressure pulse $N_{m}$ amplitude and the arc mean power $P_{d}$ on the angle $\alpha$ at $L=0,14 \mathrm{~m}$ for arc lengths $\left.l(\mathrm{~m}): 1\right)$ 0,07; 2) 0,06 ; 3 ) 0,$05 ; 4) 0,04 ; 5) 0,03$ 

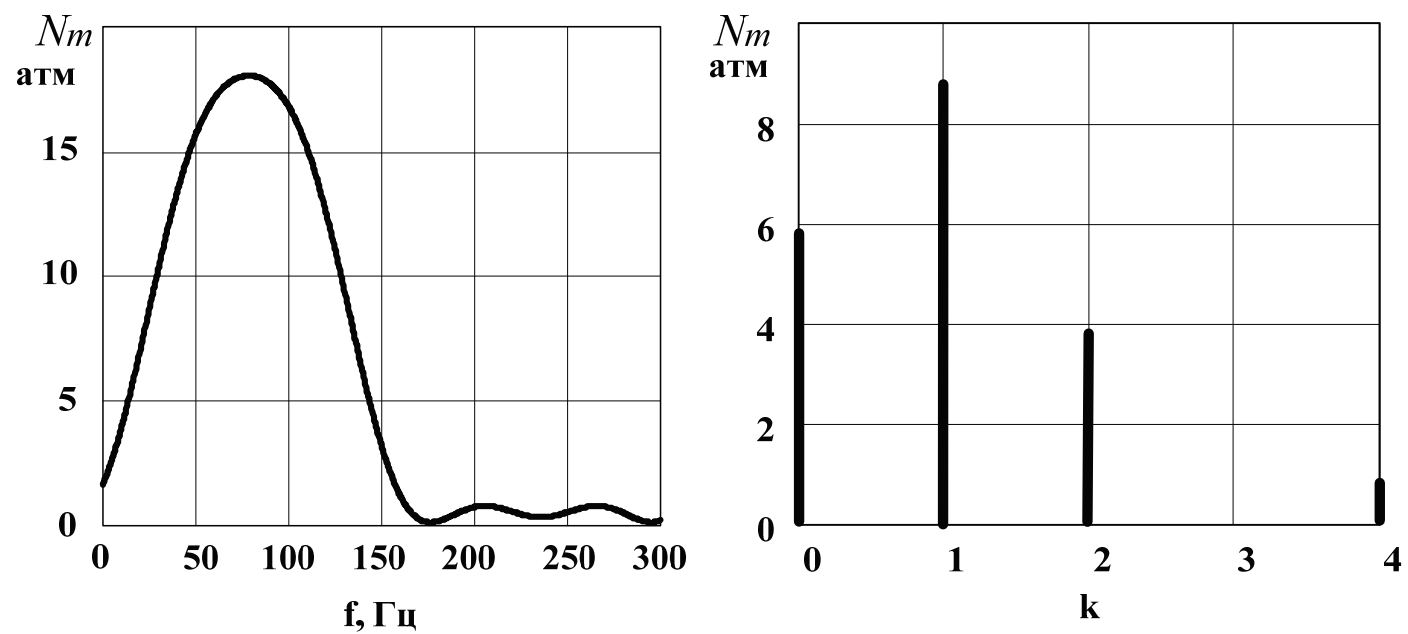

Рис. 8. Форма кривой илпульса давления $N_{m}(f)$ и алплитудно-частотный спектр илпульса давления $N_{m}(k)$

Fig. 8. Shape of the pressure pulse curve $N_{m}(f)$ and amplitude-frequency spectrum of the pressure pulse $N_{m}(k)$

здесь $\omega=314$ рад/с - угловая скорость вращения ротора ударного генератора.

На рис. 8 показана форма кривой импульса давления $N_{m}(f)$ и его амплитудно-частотный спектр $N_{m}(k)$, где $k$ - номер гармоники.

Анализ амплитудно-частотного спектра $N_{m}(k)$ показывает, что основная энергия импульса давления лежит в среднечастотном диапазоне от 50 до 100 Гц, что обеспечивает высокий сейсмический КПД для проведения сейсморазведки.

\section{Выводы}

1. Ударный генератор, выполненный в габаритах кранового асинхронного двигателя МТН-612, имеет низкое напряжение статорной обмотки $U=280$ В, что делает его применение безопасным для малоглубинной сейсморазведки при работе в полевых условиях.

2. Разработанная схема электрогидравлического излучателя с раздвигающимися электродами позволяет осуществлять инициирование электрической дуги без применения высоковольтного разряда конденсатора и взрывающихся проволочек, которые необходимо заменять после каждого взрыва.

3. Электрогидравлический излучатель с раздвигающимися электродами может осуществлять работу в режиме одиночных импульсов, а также в режиме получения серии идентичных импульсов с заданной частотой следования, что позволяет рекомендовать его применение в

\section{СПИСОК ЛИТЕРАТУРЫ}

1. Юткин Л.А. Электрогидравлический эффект и его применение в промышленности. -Л.: Изд-во «Машиностроение», 1986. - 252 с.

2. Numerical and experimental study on electrohydraulic forming process / Min-A Woo, Hong-Kyo Kim, Hyeong-Gyu Park, YongHee Kim, Woo-Jin Song, Jeong Kim // Procedia Engineering. 2017. - V. 207 - P. 311-316. электрогидравлических технологиях для электрогидравлической очистки скважин, штамповки, очистки литья и т. д.

4. Проведённые исследования с помощью математической модели работы ударного генератора на электрогидравлический излучатель с подвижными электродами показали, что наилучшими значениями независимых параметров являются: длина подвижных электродов $L=0,1-0,26 \mathrm{м}$, конечное значение длины дуги $l=0,07 \mathrm{M}$, угол включения ударного генератора $\alpha=0^{\circ}$. Максимальное значение развиваемого импульса давления $N_{m}=17,71$ атм при средней мощности дуги $N_{m}=17,71$ атм позволяет рекомендовать применение данного устройства в малоглубинной сейсморазведке на глубину до 1 км.

5. Из анализа амплитудно-частотного спектра следует, что основная энергия импульса давления лежит в среднечастотном диапазоне от 50 до 100 Гц, следовательно, предлагаемое устройство имеет высокий сейсмический КПД для проведения сейсморазведки.

6. Недостатком электрогидравлического излучателя с раздвигающимися электродами является эрозия контактов при горении дуги. Эта проблема может быть решена путём периодической замены контактов подвижных электродов, а также подбором тугоплавких материалов, увеличивающих срок службы контактов, что требует проведения дополнительных исследований.

3. Majid Ziaa, Ali Fazlia, Mahdi Soltanpoura. Warm Electrohydraulic Forming: a Novel High Speed Forming Process // Procedia Engineering. - 2017. - V. 207 - P. 323-328.

4. The research on the pulsed arc electrohydraulic discharge and its application in treatment of the ballast water / Lu Zhu, Zheng-Hao He, Pei Li, Tai-Sheng Xu, Zhi-Wen Gao // Journal of Electrostatics. - 2013. - V. 71. - Iss. 4. - P. 728-733. 
5. Research on the influence of conductivity to pulsed arc electrohydraulic discharge in water / Lu Zhu, Zheng-Hao He, ZhiWen Gao, Fa-Li Tan, Jen-Shih Chang // Journal of Electrostatics. -2014 . - V. 72. - Iss. 1. - P. 53-58.

6. Electrohydraulic shock wave generation as a means to increase intrinsic permeability of mortar / O. Maurelb, T. Reessa, M. Matallahc, A. de Ferrona, W. Chenb, C. la Borderieb, G. Pijaudier-Cabotd, A. Jacquese, F. Rey-Bethbedere // Cement and Concrete Research. - 2010. - V. 40. - Iss. 12. - P. 1631-1638.

7. Mathematical description of an asynchronous motor with the indirect control of the output mechanical variables / A.V. Glazachev, Yu.N. Dementyev, K.N. Negodin, A. Umursakova // European Physical Journal Web of Conferences (EPJ Web of Conferences). - 23 February, 2016. - V. 110. Thermophysical Basis of Energy Technologies. - 01044, 6 p. URL: http://dx.doi.org/ 10.1051/epjconf/201611001044 (дата обращения 15.10.2018).

8. Bolgov I.S., Dementiev Y.N. High-precision former of velocity and motor shaft position angle codes // Control and Communications (SIBCON): Proc. of the XII International Siberian Conference. Moscow, 12-14 May, 2016. URL: http://dx.doi.org/ 10.1109/SIBCON.2016.7491762 (дата обращения 15.10.2018).

9. Denstedt J.D., Clayman R.V. Electrohydraulic Lithotripsy of Renal and Ureteral Calculi // The Journal of Urology. - 1990. V. 143. - Iss. 1. - P. 13-17.

10. Методическое пособие по оценке размера вреда водным биоресурсам при сейсморазведке и электроразведке / В.Н. Семёнов, Ю.И. Зуенко, И.А. Атаманова, О.Н. Мухаметова, Г.С. Зеленихина, Б.В. Архипов, А.Б. Корниенко. - М.: Изд-во ВНИРО, 2016. -86 c.

11. Окунь И.З. Исследование волн сжатия, возникающих при импульсном разряде в воде // Журн. технической физики. 1971. - T. 41. - № 2. - C. 292-300.

12. Хмелевской В.К. Геофизические методы исследования земной коры. Ч. 1. - Дубна: Международный университет природы, общества и человека «Дубна», 1999. - 203 с.
13. Мирзоян Ю.Д. Источники упругой энергии и возбуждение сейсмических волн при наблюдениях ВСП на море // Геология, геофизика и разработка нефтяных и газовых месторождений. - 2000. - № 9. - С. 22-28.

14. Электроискровой источник упругих волн для целей наземной сейсморазведки / под ред. А.В. Калинина. - М.: Изд-во МГУ, 1989. $-193 \mathrm{c}$

15. 0 применении электрических машин для электрогидравлического метода сейсморазведки / Г.А. Сипайлов, К.А. Хорьков, Б.А. Франковский, Ю.Г. Шмигирилов // Силовые импульсные системы. - Новосибирск: Институт горного дела СОАН СССР, 1973. - C. $66-70$.

16. Франковский Б.А., Шмигирилов Ю.Г. Электрогидравлический источник волн давления для малоглубинной сейсморазведки // Механизация строительства. - 2015. - № 5. C. $45-48$.

17. Франковский Б.А., Шмигирилов Ю.Г. Применение электромашинного генератора для сейсморазведки // Геофизика. 2015. - № 1. - C. 82-86.

18. Pustynnikov S.V., Popov V.I., Khor'kov K.A. Mathematical model of the operation of an impact generator in an electrohydraulic device // Soviet surface engineering and applied electrochemistry. - 1989. - V. 6. - P. 57-62. URL: https://www.scopus.com/ inward/authorDetails.uri?authorID $=6508138409 \&$ partnerID=5ESL7QZV\&md5=d4d359b34614 b97e4980df15abc5797c (дата обращения 15.10.2018).

19. Кривицкий Е.В., Шамко В.В. Переходные процессы при высоковольтном разряде в воде. - Киев: Наукова думка, 1979. $208 \mathrm{c.}$

20. Сидняев Н.И. Теория планирования эксперимента и анализ статистических данных. - М.: Изд-во «Юрайт», 2016. - 496 с.

21. Копылов И.П. Проектирование электрических машин. - М.: Изд-во «Юрайт», 2017. - 767 с.

Поступила 22.10.2018 2.

\section{Информация об авторах}

Пустынников C.B., кандидат технических наук, доцент Инженерной школы электротехники Национального исследовательского Томского политехнического университета.

Носов Г.В., кандидат технических наук, доцент Инженерной школы электротехники Национального исследовательского Томского политехнического университета.

Хан Вей, кандидат технических наук, профессор Института физики, заместитель директора международного центра «Наука будущего» Цзилиньского университета.

Носова М.Г., кандидат физико-математических наук, доцент кафедры экономической математики, информатики и статистики факультета вычислительных систем Томского государственного университета систем управления и радиоэлектроники. 


\title{
INVESTIGATION OF OPERATION OF A LOW VOLTAGE SHOCK GENERATOR IN AN ELECTROHYDRAULIC DEVICE FOR SEISMIC EXPLORATION AT SMALL DEPTH
}

\section{Sergey V. Pustynnikov',}

pustynnikov@tpu.ru

Gennady V. Nosov',

nosov@tpu.ru

Wei $\mathrm{Han}^{2}$,

whan@jlu.edu.cn

\author{
Mariya G. Nosova ${ }^{3}$, \\ nosovamgm@gmail.com \\ 1 National Research Tomsk Polytechnic University, \\ 30, Lenin Avenue, Tomsk, 634050, Russia. \\ 2 Jilin University, \\ Qianin Street 2699, Changchun, 130012, P.R. China. \\ ${ }^{3}$ Tomsk State University of Control Systems and Radioelectronics, \\ 40, Lenin avenue, Tomsk, 634050, Russia.
}

The relevance of the study is caused by the need to develop new sources of powerful current pulses for operation in an electrohydraulic device with moving electrodes that can be used to excite seismic waves in non-explosive seismic surveys.

The aim of the research is to carry out computational and experimental studies of operation of a low-voltage shock generator on an arc in water, initiated by moving electrodes in the mode of single pulses and in production of a series of pressure pulses. Optimization of the parameters of the shock generator and the electrohydraulic radiator, in order to obtain the maximum pressure pulse.

Objects: low-voltage shock generator, made in the dimensions of the crane asynchronous motor MTH-612, connected to an electrohydraulic source with moving electrodes.

Methods: matching parameters of a shock generator and an arc discharge by the method of experiment planning using a mathematical model.

Results. The authors have carried out the laboratory studies of operation of a low-voltage shock generator on an arc in water initiated by moving electrodes in the regime of single pulses and in the generation of a series of pulses. Using the method of experiment planning, the authors obtained the mathematical model of the shock generator operation on an arc in water, initiated by moving electrodes in the regime of single pulses; the regression equations are obtained for output parameters of the arc discharge. Optimization of the parameters of the shock generator and the electrohydraulic source was carried out, which allows obtaining the maximum pressure pulse for seismic exploration at shallow depth. The obtained amplitude-frequency spectrum of the pressure pulse showed that the main energy of the pressure pulse lies in the low-frequency range from 0 to $180 \mathrm{~Hz}$, which provides a high seismic efficiency for seismic exploration at shallow depth.

\section{Key words:}

Shock generator, electrohydraulic source, moving electrodes, mathematical model, oscillogram, seismic survey.

\section{REFERENCES}

1. Yutkin L.A. Elektrogidravlichesky effekt $i$ ego primenenie v promyshlennosti [Electrohydraulic effect and its application in industry]. Leningrad, Mashinostroenie Publ., 1986. 252 p.

2. Min-A Woo, Hong-Kyo Kim, Hyeong-Gyu Park, Yong-Hee Kim, Woo-Jin Song, Jeong Kim. Numerical and experimental study on electrohydraulic forming process. Procedia Engineering, 2017, vol. 207, pp. 311-316.

3. Majid Ziaa, Ali Fazlia, Mahdi Soltanpoura. Warm Electrohydraulic Forming: a Novel High Speed Forming Process. Procedia Engineering, 2017, vol. 207, pp. 323-328.

4. Lu Zhu, Zheng-Hao He, Pei Li, Tai-Sheng Xu, Zhi-Wen Gao. The research on the pulsed arc electrohydraulic discharge and its application in treatment of the ballast water. Journal of Electrostatics, 2013, vol. 71, Iss. 4, pp. 728-733.

5. Lu Zhu, Zheng-Hao He, Zhi-Wen Gao, Fa-Li Tan, Jen-Shih Chang. Research on the influence of conductivity to pulsed arc electrohydraulic discharge in water. Journal of Electrostatics, 2014 , vol. 72 , Iss. 1 , pp. 53-58.
6. Maurelb 0., Reessa T., Matallahc M., De Ferrona A., Chenb W., La Borderieb C., Pijaudier-Cabotd G., Jacquese A., Rey-Bethbedere F. Electrohydraulic shock wave generation as a means to increase intrinsic permeability of mortar. Cement and Concrete Research, 2010, vol. 40, Iss. 12, pp. 1631-1638.

7. Glazachev A.V., Dementyev Yu.N., Negodin K.N., Umursakova A. Mathematical description of an asynchronous motor with the indirect control of the output mechanical variables. European Physical Journal Web of Conferences (EPJ Web of Conferences). 23 February, 2016. Vol. 110. Thermophysical Basis of Energy Technologies. 01044, 6 p. Available at: http:// dx.doi.org/10.1051/epjconf/201611001044 (accessed 15 0ctober 2018).

8. Bolgov I.S., Dementiev Y.N. High-precision former of velocity and motor shaft position angle codes. Control and Communications (SIBCON): Proc. of the XII International Siberian Conference. Moscow, 12-14 May, 2016. Available at: http:// dx.doi.org/10.1109/SIBCON.2016.7491762 (accessed 15 0ctober 2018). 
9. Denstedt J.D., Clayman R.V. Electrohydraulic Lithotripsy of Renal and Ureteral Calculi. The Journal of Urology, 1990, vol. 143, Iss. 1, pp. 13-17.

10. Semenov V.N., Zuenko Yu.I., Atamanova I.A., Mukhametova 0.N., Zelenichina G.S., Arkhipov B.V., Kornienko A.B. Metodicheskoe posobie po otsenke razmera vreda vodnym bioresursam pri seismorazvedke i elektrorazvedke [Guidelines on assessment of damage to water bioresources in seismic exploration and electrical prospecting]. Moscow, VNIR0 Publ., 2016. 86 p.

11. Okun I.Z. Issledovanie voln szhatiya, voznikayushchikh pri impulsnom razryade $\mathrm{v}$ vode [Investigation of compression waves arising during pulsed discharge in water]. Zhurnal tehhnitheskoy fiziki, 1971, vol. 41, no. 2, pp. 292-300.

12. Chmelevskiy V.K. Geofizicheskie metody issledovaniya zemnoy kory [Geophysical methods of investigation of the earth's crust]. Dubna, International University of Nature, Society and Man «Dubna» Publ., 1999. P. 1, 203 p.

13. Mirzoнan Yu.D. Istochniki uprogoy energii i vozbuzhdenie seysmicheskikh voln pri nablyudeniyakh VSP na more [Sources of elastic energy and excitation of seismic waves during observations of VSP at sea]. Geologija, geofizika i razrabotka neftjanykh i gazovykh mestorozhdeniy, 2000, no. 9, pp. 22-28.

14. Kalinin A.V. Elektroiskrovoy istochnik uprugikh voln dlya tseley nazemnoy seyismorazvedki [The spark source of elastic waves for land seismic works]. Moscow, MGU Publ., 1989. 193 p.

15. Sipaylov G.A., Khorkov K.A., Frankovskiy B.A., Shmigirilov Yu.G. 0 primenenii electrisheskich mashin dlya elektrogidra-

\section{Information about the authors}

Sergey V. Pustynnikov, Cand. Sc., associate professor, National Research Tomsk Polytechnic University.

Gennady V. Nosov, Cand. Sc., associate professor, National Research Tomsk Polytechnic University.

Wei Han, Cand. Sc., professor, Executive Deputy Director of International Center of Future Science, Jilin University.

Mariya G. Nosova, Cand. Sc., associate professor, Tomsk State University of Control Systems and Radioelectronics. vlicheskogo metoda seysmorazvedki [0n application of electrical machines for electrohydraulic seismic survey method]. Silovye impulsnye sistemy [Power impulse systems]. Novosibirsk, Institute of mining, SOAN SSSR Publ., 2008. pp. 66-70. waves for shallow seismic. Mechanization of building, 2015, no. 5, pp. 45-48. In Rus.

17. Frankovskiy B.A., Shmigirilov Yu.G. Application of an electric machine generator for seismic. Geophysics, 2015, no. 1, pp. 82-86. In Rus.

18. Pustynnikov S.V., Popov V.I., Khor'kov K.A. Mathematical model of the operation of an impact generator in an electrohydraulic device. Soviet surface engineering and applied electrochemistry, 1989, vol. 6, pp. 57-62. Available at: https://www.scopus.com/inward/authorDetails.uri?authorID $=6508138409 \&$ partner ID $=5$ ESL 7QZV \& m d $5=\mathrm{d} 4 \mathrm{~d} 359 \mathrm{~b} 34614 \mathrm{~b} 97 \mathrm{e}$ 4980df15abc5797c (accessed 15 October 2018).

19. Krivizkiji E.V., Chamko V.V. Perekhodnye protsessy pri vysokovoltnom razryade $v$ vode [Transient processes in high-voltage discharge in water]. Kiev, Naukova dumka Publ., 1979. 208 p.

20. Sindyaev N.I. Teoriya planirovaniya eksperimenta $i$ analiz statisticheskikh dannykh [Theory of experimental planning and analysis of statistical data]. Moscow, Yurayt Publ., 2016. 496 p.

21. Kopylov I.P. Proektirovanie electricheskikh machin [Designing electrical machines]. Moscow, Yurayt Publ., 2017. 767 p.

Received: 22 October 2018.
16. Frankovskiy B.A., Shmigirilov Yu.G. Electro-hudraulic pressure 